\title{
Erratum to: A Comprehensive Stylus Evaluation Methodology and Design Guidelines
}

\author{
Kanchan Jahagirdar $^{1(\bowtie)}$, Edward Raleigh ${ }^{2}$, Hanan Alnizami ${ }^{3}$, \\ Keith $\mathrm{Kao}^{2}$, and Philip J. Corriveau ${ }^{3}$ \\ ${ }^{1}$ Intel Corporation, Folsom, USA \\ kanchan. jahagirdar@intel.com \\ ${ }^{2}$ Intel Corporation, Santa Clara, USA \\ \{edward.j.raleigh, Keith.kao\}@intel.com \\ 3 Intel Corporation, Hillsboro, USA \\ \{hanan.alnizami,philip.j.corriveau\}@intel.com
}

\section{Erratum to:}

\section{Chapter 40 in: A. Marcus (Ed.)}

Design, User Experience, and Usability

DOI: 10.1007/978-3-319-40409-7_40

Figure 4c on page 430 of the paper "A Comprehensive Stylus Evaluation Methodology and Design Guidelines", authored by Kanchan Jahagirdar, Edward Raleigh, Hanan Alnizami, Keith Kao, and Philip J. Corriveau, has been retracted as it was used without permission. 